\title{
Cellular senescence: a promising strategy for cancer therapy
}

\author{
Seongju Lee ${ }^{1,2} \mathcal{E}$ Jae-Seon Lee ${ }^{1,3, *}$ \\ ${ }^{1}$ Hypoxia-related Disease Research Center, ${ }^{2}$ Department of Anatomy and ${ }^{3}$ Department of Molecular Medicine, College of Medicine, Inha \\ University, Incheon 22212, Korea
}

Cellular senescence, a permanent state of cell cycle arrest, is believed to have originally evolved to limit the proliferation of old or damaged cells. However, it has been recently shown that cellular senescence is a physiological and pathological program contributing to embryogenesis, immune response, and wound repair, as well as aging and age-related diseases. Unlike replicative senescence associated with telomere attrition, premature senescence rapidly occurs in response to various intrinsic and extrinsic insults. Thus, cellular senescence has also been considered suppressive mechanism of tumorigenesis. Current studies have revealed that therapy-induced senescence (TIS), a type of senescence caused by traditional cancer therapy, could play a critical role in cancer treatment. In this review, we outline the key features and the molecular pathways of cellular senescence. Better understanding of cellular senescence will provide insights into the development of powerful strategies to control cellular senescence for therapeutic benefit. Lastly, we discuss existing strategies for the induction of cancer cell senescence to improve efficacy of anticancer therapy. [BMB Reports 2019; 52(1): 35-41]

\section{INTRODUCTION}

Currently, cancer is the leading cause of death from illness. It is a state in which cells abnormally divide due to aberrant proliferation signals, such as oncogene activation and loss of tumor suppressors. To remove cancer cells with a strong proliferative capacity, high doses of drugs or irradiation must be administered to patients, which inevitably leads to severe side effects. As cytostasis could be an alternative way to treat cancer, cellular senescence has been suggested as a promising strategy to permanently stop proliferation of cancerous cells. In the last decade, numerous studies have sought to find the relevance between cellular senescence and cancer. It is now

${ }^{*}$ Corresponding author. Tel: +82-32-860-9832; Fax: +82-32-8858302; E-mail: jaeslee@inha.ac.kr

https://doi.org/10.5483/BMBRep.2019.52.1.294

Received 12 October 2018

Keywords: Cancer therapy, Cellular senescence, Senescence features, Senescence pathways clear that cellular senescence can act as a critical anticancer mechanism.

Cellular senescence is an irreversible cell cycle arrest that occurs in response to various forms of cellular stresses. More than five decades ago, Hayflick first described a limited ability to replicate normal human fibroblasts in culture (1). After dividing between 40 and 60 times, the human fibroblasts underwent irreversible growth arrest, however they survived for a long time, while still maintaining their metabolic activity. This state has been termed replicative senescence (RS). Telomere erosion, the gradual loss of telomeres, has been generally accepted as the cause of RS $(2,3)$. It is an intrinsic defense mechanism to avoid genomic instability, which can lead to tumorigenesis. Whereas RS has been reported in aging cells, cellular senescence has also occurred in mouse and human embryonic cells $(4,5)$. Embryonic senescence has a molecular pathway distinct from senescence in non-embryonic tissues. Senescent cells in the embryo do not express p53 and p $16^{\mathrm{INK} 4 \mathrm{a}}$, primary molecules involved in senescence occurring in non-embryonic tissues. Instead, they express p21 regulated by TGF- $\beta / S M A D$ and PI3K/FOXO pathways (4). Embryonic senescence is thought to be a regulatory mechanism to fine-tune embryogenesis (6).

Expression of oncogenes, such as RAS ${ }^{\mathrm{G} 12 \mathrm{~V}}$ or BRAF ${ }^{\mathrm{V} 600 \mathrm{E}}$, triggers oncogene-induced senescence (OIS). OIS is mediated by hyperproliferation or DNA hyper-replication and acts as an initial barrier of tumorigenesis (7). In addition to intrinsic factors, various types of extrinsic factors, such as DNA damaging agents, oxidative stress, nutrient depletion, and others cause senescence, called stress-induced premature senescence (SIPS) (8). Exposure of cancer cells to chemotherapeutic drugs or ionizing radiation also induces a senescent response, termed therapy-induced senescence (TIS) $(9,10)$. Whereas high doses of drugs or irradiation kill cancer cells by causing extensive damage, low doses primarily induce cellular senescence (11). Thus, TIS is thought to be an effective and safe way to induce cytostasis in cancer treatment, with fewer side effects than treatments that induce apoptosis.

In this review, we briefly describe key features and molecular pathways of cellular senescence. Then, we discuss current therapeutic strategies for the clinical application of cellular senescence as promising cancer therapy. 


\section{KEY FEATURES OF CELLLULAR SENESCENCE}

Several features and molecular markers have been currently used for the identification of senescent cells. However, since senescent cells are highly heterogeneous and many of their features are present in other cellular states, it is not easy to exactly identify senescent cells using only one or two markers in action $(2,12,13)$. Therefore, a comprehensive understanding of the key features of cellular senescence is essential for the clinical application of cellular senescence.

The most prominent feature of senescent cells is irreversible cell cycle arrest. Unlike quiescent cells, senescent cells do not respond to mitogens or growth factors. Senescent cells are also different from terminally differentiated cells, which also have permanently escaped cell cycle progression. Senescence is mainly caused by a cellular stress, whereas terminal differentiation is caused by a defined developmental program (14). In addition, terminally differentiated cells undergo senescence in response to various senescence-inducing stimuli. The cell cycle arrest in senescent cells is controlled by the cyclin-dependent kinase (CDK) inhibitors encoded in the CDKN2A (p16), CDKN2B (p15), and CDKN1A (p21) (14), and will be discussed in detail later.

DNA damage response (DDR) is one of the main causes of cellular senescence. Progressive telomere erosion occurring during RS ultimately exposes an uncapped, double-stranded chromosome free end detected as a double-strand break, and is a powerful activator of the DDR (15). Hyperproliferation signals induced by oncogene activation in OIS also triggers the DDR. The DDR associated with RS depends on telomeric length, whereas the DDR associated with OIS is related with telomere dysfunction $(15,16)$. Double-strand breaks recruit ATM kinase to the damaged sites, which drives phosphorylation of the histone $\mathrm{H} 2 \mathrm{AX}$, the signal for recruiting the DNA repair complex $(17,18)$. ATM also phosphorylates CHK1 and CHK2, essential kinases for propagating the signal $(19,20)$. Finally, such activated DDR machinery induces phosphorylation of p53 at multiple sites, and activated p53 induces cell cycle arrest through the transcription of downstream targets $(19,21)$. Although $\gamma-\mathrm{H} 2 \mathrm{AX}$ nuclear foci and phosphorylated p53 are commonly used as senescence markers, they have limitations in determining senescence in vivo.

Senescent cells secrete cytokines, chemokines, extracellular matrix proteases and growth factors that are termed senescence-associated secretory phenotype (SASP). The SASP is a crucial mediator of the pathological functions of senescent cells (14). Notably, senescent cells positively and negatively regulate tumorigenesis and the immune response of their neighbors via the SASP. For example, the SASP from senescent human fibroblasts induces paracrine senescence in neighboring normal cells (22). On the contrary, the SASP can also promote tumorigenesis. The SASP of senescent hepatic stellate cells promotes the proliferation and malignancy of the neighboring cells in obese mice exposed to chemical carcinogens (23). The
SASP is regulated at multiple levels. Transcriptional factors involved in SASP regulation are NF-KB, mitochondrial dysfunction-associated senescence (MiDAS), GATA4, and CCAAT/enhancer-binding protein- $\beta$ (C/EBP $\beta)$ (24-27). In particular, NF- $\mathrm{\kappa B}$ and $\mathrm{C} / \mathrm{EBP} \beta$ mainly regulate the SASP by directly controlling key regulators of the inflammatory SASP, such as IL-6 and IL-8 $(27,28)$. The SASP is also regulated by mTOR at the post-transcriptional level. mTOR mediates translation of IL-1A and MAP kinase-activated protein kinase 2 (MAPKAPK2) $(29,30)$. In addition, the SASP is regulated at an epigenetic level via SIRT1, macroH2A1, and MLL1 (31-33). For example, a decrease in SIRT1, a histone deacetylase, in senescent cells results in an increase of IL-6 and IL-8 expression by histone acetylation at their promoter regions (31).

Senescence is generally accompanied by significant morphological alterations. The senescent cells become flat and enlarged due to rearrangement of the cytoskeleton. Their plasma membrane composition is changed by the upregulation of caveolin-1, a main component of caveolae (34, 35). Senescent cells also have an increased cholesterol content in membrane and large and dysfunctional mitochondria (34, 35). Lysosomal content and lysosomal enzyme senescenceassociated $\beta$-galactosidase (SA- $\beta$-gal) is highly upregulated during senescence (36). Thus, the activity of SA- $\beta$-gal is most widely used to indicate senescence. Lamin B1, a structural protein of the nuclear lamina, is commonly reduced in senescent cells (37). Formation of senescence-associated heterochromatic foci (SAHFs) is another prominent feature of senescence. The foci are characterized by enrichment of repressive epigenetic marks, such as methylated H3K9, heterochromatic protein 1 (HP1), and macroH2A (38).

\section{MOLECULAR PATHWAYS CONTROLLING CELLLULAR SENESCENCE}

The senescent cell cycle arrest is mainly controlled by two interconnected pathways: activation of the p53/p21 ${ }^{\mathrm{CIP} 1}$ and p16 $16^{\text {INK4a }} /$ RB tumor suppressor pathways (Fig. 1). Telomere erosion or OIS activates p53 through DDR, while PTEN loss-induced senescence (PICS) activates p53 through the mTOR pathway $(14,39)$. DDR transduced by ATMCHK2 and ATR/CHK1 blocks cell cycle progression via phosphorylation and stabilization of p53 $(2,11,14)$. Activation of p53 promotes transcription of the CDK inhibitor $\mathrm{p} 21^{\mathrm{CIP} 1}$ encoded by $C D K N 1 A$, which inhibits CDK2 activity, thereby activating $\mathrm{RB}$ and inducing cell cycle arrest (19). The CDKN2A locus consists of $\mathrm{p} 14^{\text {ARF }}, \mathrm{p} 16^{\text {INK4a }}$, and $\mathrm{p} 15^{\mathrm{INK} 4 \mathrm{~B}}$, encoding key regulators of cellular senescence (40). In proliferating cells, the locus is expressed at a very low level due to binding of the repressive complexes, PRC1 and PRC2 (41). During senescence, gene expression from CDKN2A locus gradually increases (42). p14 ${ }^{\mathrm{ARF}}$ prevents p53 degradation by degrading MDM2 proto-oncogene. Meanwhile, $\mathrm{p} 16^{\text {INK4a }}$ and $\mathrm{p} 15^{\text {INK4B }}$ 


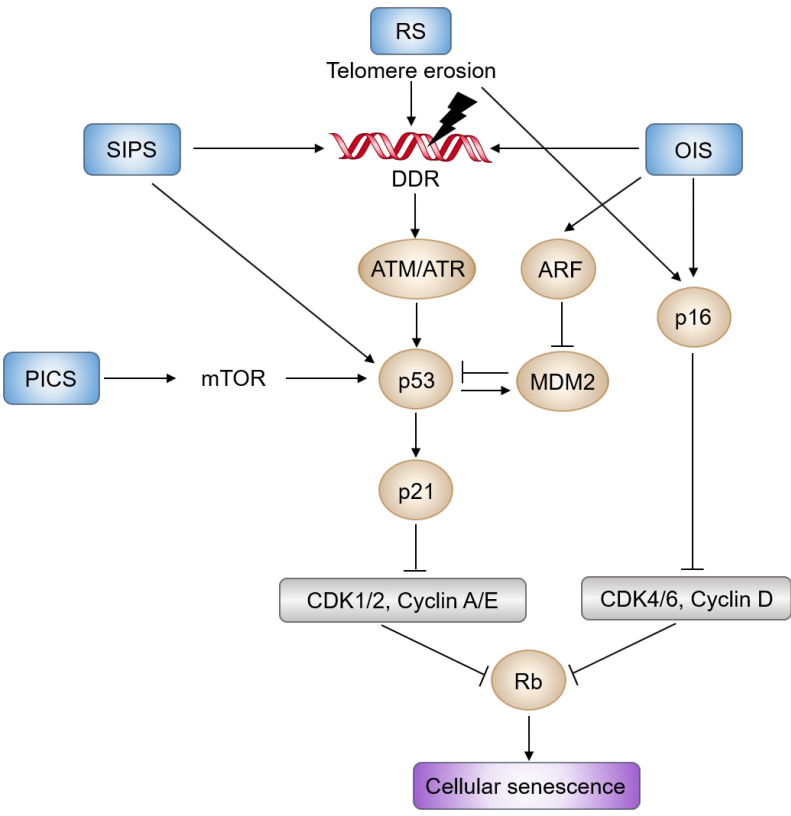

Fig. 1. Molecular pathways of cellular senescence. DDR (DNA damage response) triggered by telomere erosion or OIS (oncogeneinduced senescence) is mediated by ATM/CHK2 and ATR/CHK1, which blocks cell cycle progression via phosphorylation and stabilization of p53. Activated p53 induces transcription of CDK inhibitor p21 ${ }^{\mathrm{CIP1}}$ encoded by $C D K N 1 A$, which inhibits CDK2 activity, thereby activating RB and inducing cell cycle arrest. In parallel, various stressors also induce expression of the CDKN2A locus, which consists of $\mathrm{p} 14^{\mathrm{ARF}}, \mathrm{p} 16^{\mathrm{INK} 4 \mathrm{a}}$, and $\mathrm{p} 15^{\mathrm{NK} 4 \mathrm{~B}}$. $\mathrm{p} 14^{\mathrm{ARF}}$ prevents p53 destabilization by degrading the MDM2 protooncogene. Meanwhile, p16 ${ }^{\text {INK4a }}$ and $\mathrm{p} 15^{\text {INK4B }}$ suppress CDK4 and CDK6, thereby eventually activating RB. PICS (PTEN loss-induced cellular senescence) also activates p53 through the mTOR pathway. Altogether, prolonged activation of the $\mathrm{p} 53 / \mathrm{p} 21^{\mathrm{CIP} 1}$ and p16 $6^{\text {INK4a }} /$ RB signaling pathways leads to hypophosphorylation of RB, which blocks the cell cycle in G1 phase, and ultimately causes cellular senescence. RS and SIPS indicate replicative senescence and stress-induced premature senescence, respectively.

selectively suppress CDK4 and CDK6, causing the eventual activation of RB. Altogether, stress-induced activation of the $\mathrm{p} 53 / \mathrm{p} 21^{\mathrm{CIP} 1}$ and $\mathrm{p} 16^{\mathrm{INK} 4 \mathrm{a}} / \mathrm{RB}$ pathways leads to hypophosphorylation of RB, which blocks the cell cycle in G1/G2 phase, and ultimately causes cellular senescence.

Senescent cells generally exhibit enhanced glycolysis (43). Thus, senescent cells increase the ratio of adenosine diphosphate (ADP) and adenosine monophosphate (AMP), relative to adenosine triphosphate (ATP), which activates AMP-activated protein kinase (AMPK), a master regulator of energy stress. Activated AMPK directly phosphorylates p53, which upregulates transcription of $\mathrm{p} 21^{\mathrm{CIP} 1}$ (44). On the other hand, AMPK increases the stability of $\mathrm{p} 21^{\text {waf1 }}$ and $\mathrm{p} 16^{\text {INK4a }}$ mRNAs by inhibiting $\mathrm{Hu}$ antigen R-mediated degradation, finally enhancing RB activity (45). Through these two mechanisms, AMPK regulates cell cycle arrest, and consequently cellular senescence.

Recently, an insulin-like growth factor 1 (IGF-1)/sirtuin-1 (SIRT1)/p53 pathway involved in cellular senescence was identified $(46,47)$. The SIRT1 plays a crucial role in cellular senescence as well as in aging. SIRT1 level is reduced in senescent human fibroblasts, and inhibition of SIRT1 by sirtinol induces senescence response in cancer cells $(48,49)$. Conversely, overexpression of SIRT1 antagonizes OIS (50). Among seven human sirtuin family proteins (SIRT1 through 7) sharing the catalytic domain with yeast Sir2, only SIRT1 can deacetylate p53, resulting in inactivation of p53 $(48,51,52)$. IGF signaling generally plays a mitogenic role $(47,53)$. Specifically, activation of IGF1R upregulates the PI3KJAKT/mTOR pathway, thereby promoting growth and proliferation. Interestingly, acute exposure of IGF-1 to human fibroblasts promotes cell proliferation and survival, whereas prolonged administration of IGF-1 induces premature senescence (46, 47). Mechanistically, prolonged IGF-1 treatment inhibits the activity of SIRT1, which results in increased acetylation and activation of p53, thereby leading to premature senescence (46). It was recently revealed that mTOR phosphorylates p53 through direct binding, resulting in cellular senescence, under PICS and AKT-activated conditions (54).

Deregulation of autophagy, a process for lysosomal degradation and recycling of intracellular components, has been linked to various human diseases, ranging from cardiomyopathy to neurodegeneration $(55,56)$. Since autophagy is another stress response essential for homeostasis, autophagy and senescence share a number of common characteristics. Although an increase in autophagy has been observed in senescent cells, their relationship remains poorly defined $(14,57,58)$. At the beginning of these studies, a positive feedback loop between autophagy and senescence seemed to be existed. For instance, expression of RAS ${ }^{\mathrm{G} 12 \mathrm{~V}}$ induces autophagic activity, and expression of autophagic genes, such as ULK3, induces senescence (59). Moreover, inhibition of autophagy delays the senescence response (59, 60). Conversely, some studies have shown that inhibition of autophagy facilitates senescence $(26,61)$. Depletion of autophagic adaptors, such as p62, induces senescence. The paradox might be explained by the autophagic degradation of GATA4 (26). GATA4 is a transcription factor essential for regulation of the SASP. During senescence, GATA4 degradation by p62-mediated selective autophagy is suppressed, thereby resulting in GATA4 stabilization, which is a favorable state for SASP production. Taken together, these studies suggest that selective autophagy inhibits senescence, but that general autophagy supports senescence.

\section{INDUCING SENESCENCE: PROMISING STRATEGY FOR CANCER THERAPY}

Traditional cancer therapy aims to kill rapidly dividing cancer cells by inducing extensive DNA damage using high doses of 
drugs or irradiation. The strategy is effective and powerful, but causes severe side effects, such as neighboring normal cell damage, cancer recurrence, and cancer cell resistance to therapy. As described earlier, TIS, a senescent response caused by conventional cancer therapy, is suggested as an alternative and effective way for cancer treatment. In addition, a large number of studies support that SIPS acts as a barrier in cancer progression in vitro and in vivo $(7,62)$. Therefore, drug discovery which selectively induces senescence in cancer cells could represent a promising approach for cancer intervention, through a process called pro-senescence therapy $(7,39,63)$. In this section, we review the latest approaches to induce senescence in cancer cells and their underlying rationale.

\section{Modulating tumor suppressors}

p53, the most frequently mutated tumor suppressor in cancer, is an important effector of cellular senescence. Recent studies emphasize that the p53/p21 axis is a promising target for pro-senescence therapy $(39,64-69)$. Since p53 is stabilized and accumulated in senescent cells, researchers made an effort to develop small molecules to increase the amount or activity of p53 in cancer cells. One of the initially discovered small molecules, Nutlin, stabilizes p53 by inhibiting the interaction of p53 with MDM2 (66). A study using mouse models of PICS indicates that administration of Nutlin-3 significantly increases the p53 protein level and inhibits tumorigenesis in vivo (67). Inhibition of the interaction between p53 and MDM4, a negative regulator of p53, restores p53 activity in melanoma cells and results in increased sensitivity to cytostatic or cytotoxic chemotherapy (68). The restoration of p53 in murine premalignant proliferating p53-deficient cells also induces senescence and halts further proliferation in vitro and in vivo (69). In addition, FOXO4 is identified as pivot in senescent cell viability and FOXO4 peptide that perturbs p53-FOXO4 interaction is developed (70). FOXO4 peptide leads to nuclear exclusion of p53 and cell-intrinsic apoptosis in senescent cells. Administration of $\mathrm{FOXO} 4$ peptide neutralizes liver chemotoxicity and restores fitness, hair density, and renal function in fast aging and naturally aged mice. Recently, it has been shown that undersulfation of heparan sulfate proteoglycan induces augmentation of fibroblast growth factor receptor 1 (FGFR1) signaling, ultimately resulting in premature senescence through p53 activation and retards tumor growth in a xenograft tumor mouse model (71). Modulation of JNK activity also effectively activates p53 via regulation of $\mathrm{Bcl}-2$ phosphorylaiton and ROS generation, and finally resulted in cellular senescence (72).

Phosphatase and tensin homolog (PTEN) deleted on chromosome 10 catalyzes the conversion of PIP3 to PIP2, and functions as a key negative regulator of the AKT/PKB signaling pathway (73). PTEN is another most frequently altered tumor suppressor in cancer, particularly prostate cancer (74). Thus, PTEN dose is a critical determinant in cancer progression. Heterozygous loss of PTEN causes tumor development, whereas complete loss of PTEN triggers non-lethal invasive cancer after a long latency, through the p53-mediated senescence pathway (75). In addition, PTEN is suggested to be a pivotal determinant of cell fate between senescence and apoptosis in glioma cells exposed to ionizing radiation (76). Therefore, inactivation of PTEN in cancer cells would be an attractive way of pro-senescence therapy. Treatment of VO-OHpic, a PTEN inhibitor, in Pten $^{+/-}$MEFs induces senescence through hyperactivation of a AKT-mTOR-p53 pathway $(67,72)$. A recent chemogenomic screening in PTEN-deficient cells revealed casein kinase 2 (CK2) as a pro-senescent target (77). PTEN-deficiency increases CK2 levels, resulting in stabilization of $\mathrm{Pml}$, a crucial regulator of senescence. Application of CK2 inhibitors such as Quinalizarin and CX-4945 enhances PICS and blocks tumor progression under PTEN-deficient conditions.

\section{Targeting cell cycle machinery}

The activity of several cell cycle kinases including CDKs is often deregulated in cancer cells. Over the last thirty years, many drugs have been identified as CDK inhibitors through intensive searches, although these CDK inhibitors have failed as anti-cancer drugs, due to toxicity and limited activity (78). However, recent studies indicate that administration of CDK inhibitors in a specific genetic background can cause a tumor-specific senescence (79-81). Pharmacological inhibition of CDK2, such as CVT-313 and CVT-2584, in the context of c-Myc overexpression leads to senescence induction (79). Genetic ablation of CDK4 also induces senescence response and tumor regression in K-RAS ${ }^{\mathrm{G} 12 \mathrm{~V}}$ expressing lung cells (80). Administration of a selective CDK4 inhibitor, PD0332991, results in similar effects on lung tumors driven by K-RAS ${ }^{\mathrm{G} 12 \mathrm{~V}}$. The E3 ubiquitin ligase S-phase kinase-associated protein 2 (SKP2) regulates cell cycle via p27 degradation. Recent work reported that genetic inactivation of SKP2 induces senescence and suppresses tumorigenesis, partly through p27 accumulation, even in a p53-impaired condition (81). Given that pharmacological inhibition of SKP2 by MLN4924 is effective, SKP2 may be a potential target for cancer therapy.

\section{Manipulating the SASP}

As described above, the SASP can have various and opposite effects on neighboring normal and cancer cells. Secreted SASP factors could reinforce stable cell cycle arrest and suppress tumorigenesis, by signaling to and recruiting the immune system $(82,83)$. Therefore, the secreted factors themselves can be used as prototypal drugs (7). Long term treatment with SASP constituents, such as plasminogen activator inhibitor 1 (PAI1), interleukin-6 (IL-6), interleukin-8 (IL-8), insulin-like growth factor binding protein 7 (IGFBP7), and transforming growth factor- $\beta$ (TGF- $\beta$ ) could induce senescence in cancer cells. However, the SASP also has opposing roles for antitumorigenic effects, depending on the genetic context. In addition, secreted SASPs promote tumorigenesis by promoting 
angiogenesis through VEGF production or by inducing the epithelial-to-mesenchymal transition of cancerous cells (84-86). Therefore, well-defined manipulation of SASP constituent is necessary to develop further promising way of pro-senescent therapy.

\section{CONCLUSION}

When it was discovered fifty years ago, cellular senescence was simply considered to be an artifact phenomenon. However, a multitude of studies have revealed that senescence exists as a possible defense mechanism to remove irreparable cellular injury. In addition, recent studies have revealed that cellular senescence can provide an alternative approach that can overcome the limitations of conventional cancer therapy. Indeed, several molecules that could be used in prosenescence therapy are currently undergoing clinical trials. More recently, efforts to develop senolytic drugs that can induce cell death in senescent cells are increasing. In particular, senolytic drugs could be combined with pro-senescence therapy, in case of that immune system cannot clear senescent cells effectively. This combination strategy would lower the risk of side effects from pro-senescence therapy alone. It should not be overlooked, however, that the TIS has a dark side as well. This strategy can often behave as a double-edged sword because it has opposing effects on cell proliferation and tumorigenesis, depending on the genetic context. For example, oncogenic Ras either behaves as a potent oncogene or halts proliferation depending on expression levels or genetic context. And the SASP, such as IL-6 and IL-8 can be either tumor suppressive or protumorigenic. Therefore, pro-senescence therapy should be applied with caution. Overall, in spite of some defects, we believe that pro-senescence therapy is expected as a powerful and promising strategy for anticancer therapy.

\section{ACKNOWLEDGEMENTS}

This work was supported by grants (MRC 2014R1A5A2 009392, 2017R1A2B2007542, 2017M2A2A7A01070591, and 2016R1A6A3A04006478) through the National Research Foundation (NRF) funded by the Korean government (MSIT).

\section{CONFLICTS OF INTEREST}

The authors have no conflicting interests.

\section{REFERENCES}

1. Hayflick $L$ (1965) The limited in vitro lifetime of human diploid cell strains. Exp Cell Res 37, 614-636

2. Campisi J (2013) Aging, cellular senescence, and cancer. Annu Rev Physiol 75, 685-705

3. Deng $Y$ and Chang S (2007) Role of telomeres and telomerase in genomic instability, senescence and cancer. Lab Invest 87, 1071-1076

4. Munoz-Espin D, Canamero M, Maraver A et al (2013) Programmed cell senescence during mammalian embryonic development. Cell 155, 1104-1118

5. Storer M, Mas A, Robert-Moreno A et al (2013) Senescence is a developmental mechanism that contributes to embryonic growth and patterning. Cell 155, 1119-1130

6. Campisi J (2014) Cell biology: The beginning of the end. Nature 505, 35-36

7. Acosta JC and Gil J (2012) Senescence: a new weapon for cancer therapy. Trends Cell Biol 22, 211-219

8. Itahana K, Campisi J and Dimri GP (2004) Mechanisms of cellular senescence in human and mouse cells. Biogerontology 5, 1-10

9. Chang BD, Broude EV, Dokmanovic M et al (1999) A senescence-like phenotype distinguishes tumor cells that undergo terminal proliferation arrest after exposure to anticancer agents. Cancer Res 59, 3761-3767

10. Chang BD, Xuan Y, Broude EV et al (1999) Role of p53 and $\mathrm{p} 21$ waf1/cip1 in senescence-like terminal proliferation arrest induced in human tumor cells by chemotherapeutic drugs. Oncogene 18, 4808-4818

11. Lee M and Lee JS (2014) Exploiting tumor cell senescence in anticancer therapy. BMB Rep 47, 51-59

12. Hernandez-Segura A, Nehme J and Demaria M (2018) Hallmarks of Cellular Senescence. Trends Cell Biol 28, 436-453

13. Myrianthopoulos V, Evangelou K, Vasileiou PVS et al (2019) Senescence and senotherapeutics: a new field in cancer therapy. Pharmacol Ther 193, 31-49

14. Herranz N and Gil J (2018) Mechanisms and functions of cellular senescence. J Clin Invest 128, 1238-1246

15. d'Adda di Fagagna F, Reaper PM, Clay-Farrace L et al (2003) A DNA damage checkpoint response in telomereinitiated senescence. Nature 426, 194-198

16. Suram A, Kaplunov J, Patel PL et al (2012) Oncogeneinduced telomere dysfunction enforces cellular senescence in human cancer precursor lesions. EMBO J 31, 2839-2851

17. Shiloh Y (2006) The ATM-mediated DNA-damage response: taking shape. Trends Biochem Sci 31, 402-410

18. Celeste A, Petersen S, Romanienko PJ et al (2002) Genomic instability in mice lacking histone $\mathrm{H} 2 \mathrm{AX}$. Science 296, 922-927

19. d'Adda di Fagagna F (2008) Living on a break: cellular senescence as a DNA-damage response. Nat Rev Cancer $8,512-522$

20. Lukas C, Falck J, Bartkova J, Bartek J and Lukas J (2003) Distinct spatiotemporal dynamics of mammalian checkpoint regulators induced by DNA damage. Nat Cell Biol 5, 255-260

21. Turenne GA, Paul P, Laflair L and Price BD (2001) Activation of p53 transcriptional activity requires ATM's kinase domain and multiple $\mathrm{N}$-terminal serine residues of p53. Oncogene 20, 5100-5110

22. Acosta JC, Banito A, Wuestefeld T et al (2013) A complex secretory program orchestrated by the inflammasome controls paracrine senescence. Nat Cell Biol 15, 978-990

23. Yoshimoto S, Loo TM, Atarashi K et al (2013) Obesity- 
induced gut microbial metabolite promotes liver cancer through senescence secretome. Nature 499, 97-101

24. Ohanna M, Giuliano S, Bonet $C$ et al (2011) Senescent cells develop a PARP-1 and nuclear factor-KB-associated secretome (PNAS). Genes Dev 25, 1245-1261

25. Wiley CD, Velarde MC, Lecot $P$ et al (2016) Mitochondrial dysfunction induces senescence with a distinct secretory phenotype. Cell Metab 23, 303-314

26. Kang C, Xu Q, Martin TD et al (2015) The DNA damage response induces inflammation and senescence by inhibiting autophagy of GATA4. Science 349, aaa5612

27. Kuilman T and Peeper DS (2008) Oncogene-induced senescence relayed by an interleukin-dependent inflammatory network. Cell 133, 1019-1031

28. Acosta JC, O'Loghlen A, Banito A et al (2008) Chemokine signaling via the CXCR2 receptor reinforces senescence. Cell 133, 1006-1018

29. Laberge RM, Sun Y, Orjalo AV et al (2015) MTOR regulates the pro-tumorigenic senescence-associated secretory phenotype by promoting IL1A translation. Nat Cell Biol 17, 1049-1061

30. Herranz N, Gallage S, Mellone M et al (2015) mTOR regulates MAPKAPK2 translation to control the senescenceassociated secretory phenotype. Nat Cell Biol 17, 12051217

31. Hayakawa T, Iwai M, Aoki S et al (2015) SIRT1 suppresses the senescence-associated secretory phenotype through epigenetic gene regulation. PLoS One 10, e0116480

32. Chen H, Ruiz PD, McKimpson WM et al (2015) MacroH2A1 and ATM play opposing roles in paracrine senescence and the senescence-associated secretory phenotype. Mol Cell 59, 719-731

33. Capell BC, Drake AM, Zhu J et al (2016) MLL1 is essential for the senescence-associated secretory phenotype. Genes Dev 30, 321-336

34. Jung $\mathrm{SH}$, Lee $M$, Park $\mathrm{HA}$ et al (2018) Integrin $\alpha 6 \beta 4-S r c-A K T$ signaling induces cellular senescence by counteracting apoptosis in irradiated tumor cells and tissues. Cell Death Differ 26, 245-259

35. Ohno-Iwashita $Y$, Shimada $Y$, Hayashi $M$ and Inomata $M$ (2010) Plasma membrane microdomains in aging and disease. Geriatr Gerontol Int 10, S41-52

36. Kurz DJ, Decary S, Hong Y and Erusalimsky JD (2000) Senescence-associated $\beta$-galactosidase reflects an increase in lysosomal mass during replicative ageing of human endothelial cells. J Cell Sci 113, 3613-3622

37. Sadaie M, Salama R, Carroll T et al (2013) Redistribution of the Lamin B1 genomic binding profile affects rearrangement of heterochromatic domains and SAHF formation during senescence. Genes Dev 27, 1800-1808

38. Salama R, Sadaie M, Hoare M and Narita M (2014) Cellular senescence and its effector programs. Genes Dev 28, 99-114

39. Nardella C, Clohessy JG, Alimonti A and Pandolfi PP (2011) Pro-senescence therapy for cancer treatment. Nat Rev Cancer 11, 503-511

40. Kim WY and Sharpless NE (2006) The regulation of INK4/ARF in cancer and aging. Cell 127, 265-275

41. Bracken AP, Kleine-Kohlbrecher $D$, Dietrich $N$ et al (2007) The Polycomb group proteins bind throughout the
INK4A-ARF locus and are disassociated in senescent cells. Genes Dev 21, 525-530

42. Alcorta DA, Xiong Y, Phelps D, Hannon G, Beach D and Barrett JC (1996) Involvement of the cyclin-dependent kinase inhibitor p16 (INK4a) in replicative senescence of normal human fibroblasts. Pro Natl Acad Sci U S A 93, 13742-13747

43. Wiley CD and Campisi J (2016) From ancient pathways to aging cells-connecting metabolism and cellular senescence. Cell Metab 23, 1013-1021

44. Jones RG, Plas DR, Kubek S et al (2005) AMP-activated protein kinase induces a p53-dependent metabolic checkpoint. Mol Cell 18, 283-293

45. Wang W, Yang X, Lopez de Silanes I, Carling D and Gorospe M (2003) Increased AMP:ATP ratio and AMP-activated protein kinase activity during cellular senescence linked to reduced HuR function. J Biol Chem 278, 27016-27023

46. Tran D, Bergholz J, Zhang $\mathrm{H}$ et al (2014) Insulin-like growth factor-1 regulates the SIRT1-p53 pathway in cellular senescence. Aging Cell 13, 669-678

47. Angelini F, Pagano F, Bordin A et al (2017) Getting old through the blood: Circulating molecules in aging and senescence of cardiovascular regenerative cells. Front Cardiovasc Med 4, 62

48. Michishita E, Park JY, Burneskis JM, Barrett JC and Horikawa I (2005) Evolutionarily conserved and nonconserved cellular localizations and functions of human SIRT proteins. Mol Biol Cell 16, 4623-4635

49. Ota H, Tokunaga E, Chang K et al (2006) Sirt1 inhibitor, Sirtinol, induces senescence-like growth arrest with attenuated Ras-MAPK signaling in human cancer cells. Oncogene 25, 176-185

50. Huang J, Gan Q, Han L et al (2008) SIRT1 overexpression antagonizes cellular senescence with activated ERK/S6k1 signaling in human diploid fibroblasts. PLoS One 3, e1710

51. Langley E, Pearson M, Faretta M et al (2002) Human SIR2 deacetylates p53 and antagonizes PMLp53-induced cellular senescence. EMBO J 21, 2383-2396

52. Ong ALC and Ramasamy TS (2018) Role of Sirtuin1-p53 regulatory axis in aging, cancer and cellular reprogramming. Ageing Res Rev 43, 64-80

53. Blume-Jensen $P$ and Hunter T (2001) Oncogenic kinase signalling. Nature 411, 355-365

54. Jung SH, Hwang HJ, Kang D et al (2018) mTOR kinase leads to PTEN-loss-induced cellular senescence by phosphorylating p53. Oncogene [Epub ahead of print]

55. Schneider JL and Cuervo AM (2014) Autophagy and human disease: emerging themes. Curr Opin Genet Dev 26, 16-23

56. Chang J, Lee $S$ and Blackstone C (2014) Spastic paraplegia proteins spastizin and spatacsin mediate autophagic lysosome reformation. J Clin Invest 124, 5249-5262

57. Gewirtz DA (2013) Autophagy and senescence: A partnership in search of definition. Autophagy 9, 808

58. Kang C and Elledge SJ (2016) How autophagy both activates and inhibits cellular senescence. Autophagy 12, 898-899

59. Young AR, Narita M, Ferreira M et al (2009) Autophagy 
mediates the mitotic senescence transition. Genes Dev 23, 798-803

60. Mosieniak G, Adamowicz M, Alster O et al (2012) Curcumin induces permanent growth arrest of human colon cancer cells: link between senescence and autophagy. Mech Ageing Dev 133, 444-455

61. Wang Y, Wang XD, Lapi E et al (2012) Autophagic activity dictates the cellular response to oncogenic RAS. Proc Natl Acad Sci U S A 109, 13325-13330

62. Kim BC, Yoo HJ, Lee HC et al (2014) Evaluation of premature senescence and senescence biomarkers in carcinoma cells and xenograft mice exposed to single or fractionated irradiation. Oncol Rep 31, 2229-2235

63. Ewald JA, Desotelle JA, Wilding G and Jarrard DF (2010) Therapy-induced senescence in cancer. J Natl Cancer Inst $102,1536-1546$

64. Ovadya Y and Krizhanovsky V (2018) Strategies targeting cellular senescence. J Clin Invest 128, 1247-1254

65. Yosef R, Pilpel N, Papismadov N et al (2017) p21 maintains senescent cell viability under persistent DNA damage response by restraining JNK and caspase signaling. EMBO J 36, 2280-2295

66. Vassilev LT, Vu BT, Graves B et al (2004) In vivo activation of the p53 pathway by small-molecule antagonists of MDM2. Science 303, 844-848

67. Alimonti A, Nardella C, Chen Z et al (2010) A novel type of cellular senescence that can be enhanced in mouse models and human tumor xenografts to suppress prostate tumorigenesis. J Clin Invest 120, 681-693

68. Gembarska A, Luciani F, Fedele C et al (2012) MDM4 is a key therapeutic target in cutaneous melanoma. Nat Med $18,1239-1247$

69. Harajly M, Zalzali H, Nawaz Z et al (2016) p53 restoration in induction and maintenance of senescence: Differential effects in premalignant and malignant tumor cells. Mol Cell Biol 36, 438-451

70. Baar MP, Brandt RMC, Putavet DA et al (2017) Targeted apoptosis of senescent cells restores tissue homeostasis in response to chemotoxicity and aging. Cell 169, 132-147

71. Jung SH, Lee HC, Yu DM et al (2016) Heparan sulfation is essential for the prevention of cellular senescence. Cell Death Differ 23, 417-429

72. Lee JJ, Lee JH, Ko YG, Hong SI and Lee JS (2010) Prevention of premature senescence requires JNK regulation of Bcl-2 and reactive oxygen species. Oncogene 29, 561-575

73. Stambolic V, Suzuki A, de la Pompa JL et al (1998)
Negative regulation of $\mathrm{PKB} / \mathrm{Akt}$-dependent cell survival by the tumor suppressor PTEN. Cell 95, 29-39

74. Trotman LC, Niki M, Dotan ZA et al (2003) Pten dose dictates cancer progression in the prostate. PLoS Biol 1, E5

75. Chen Z, Trotman LC, Shaffer D et al (2005) Crucial role of p53-dependent cellular senescence in suppression of Pten-deficient tumorigenesis. Nature 436, 725-730

76. Lee JJ, Kim BC, Park MJ et al (2011) PTEN status switches cell fate between premature senescence and apoptosis in glioma exposed to ionizing radiation. Cell Death Differ 18, 666-677

77. Kalathur M, Toso A, Chen J et al (2015) A chemogenomic screening identifies CK2 as a target for pro-senescence therapy in PTEN-deficient tumours. Nat Commun 6, 7227

78. Lapenna S and Giordano A (2009) Cell cycle kinases as therapeutic targets for cancer. Nat Rev Drug Discov 8, 547-566

79. Campaner S, Doni M, Hydbring P et al (2010) Cdk2 suppresses cellular senescence induced by the c-myc oncogene. Nat Cell Biol 12, 54-59

80. Puyol M, Martin A, Dubus P et al (2010) A synthetic lethal interaction between K-Ras oncogenes and Cdk4 unveils a therapeutic strategy for non-small cell lung carcinoma. Cancer Cell 18, 63-73

81. Lin HK, Chen Z, Wang G et al (2010) Skp2 targeting suppresses tumorigenesis by Arf-p53-independent cellular senescence. Nature 464, 374-379

82. Kuilman T and Peeper DS (2009) Senescence-messaging secretome: SMS-ing cellular stress. Nat Rev Cancer 9, 81-94

83. Coppe JP, Desprez PY, Krtolica A and Campisi J (2010) The senescence-associated secretory phenotype: the dark side of tumor suppression. Annu Rev Pathol 5, 99-118

84. Coppe JP, Kauser K, Campisi J and Beausejour CM (2006) Secretion of vascular endothelial growth factor by primary human fibroblasts at senescence. J Biol Chem 281, 29568-29574

85. Coppe JP, Patil CK, Rodier F et al (2008) Senescenceassociated secretory phenotypes reveal cell-nonautonomous functions of oncogenic RAS and the p53 tumor suppressor. PLoS Biol 6, 2853-2868

86. Han NK, Kim BC, Lee HC et al (2012) Secretome analysis of ionizing radiation-induced senescent cancer cells reveals that secreted RKIP plays a critical role in neighboring cell migration. Proteomics 12, 2822-2832 